\title{
Comparison between the Homotopy Perturbation Method and Homotopy Perturbation Transform Method
}

\author{
Mohamed Elbadri1,2 \\ ${ }^{1}$ Department of Mathematics, Al Jouf University, Tabarjal, KSA \\ ${ }^{2}$ Department of Mathematics, University of Gezira, Wad Madani, Sudan \\ Email: badry19822@gmail.com
}

How to cite this paper: Elbadri, M. (2018) Comparison between the Homotopy Perturbation Method and Homotopy Perturbation Transform Method. Applied Mathematics, 9, 130-137.

https://doi.org/10.4236/am.2018.92009

Received: January 3, 2018

Accepted: February 20, 2018

Published: February 23, 2018

Copyright $\odot 2018$ by author and Scientific Research Publishing Inc. This work is licensed under the Creative Commons Attribution International License (CC BY 4.0).

http://creativecommons.org/licenses/by/4.0/

\section{c) (i) Open Access}

\begin{abstract}
In this paper, comparison of homotopy perturbation method (HPM) and homotopy perturbation transform method (HPTM) is made, revealing that homotopy perturbation transform method is very fast convergent to the solution of the partial differential equation. For illustration and more explanation of the idea, some examples are provided.
\end{abstract}

\section{Keywords}

Homotopy Perturbation Method (HPM), Laplace Transform

\section{Introduction}

Many problems in our life are modeled by linear and nonlinear partial differential equations. In recent years, various analytical methods proposed by researchers to solve these PDEs. However, it is still very difficult to obtain closed-form solutions. The homotopy perturbation method proposed by Ji-Huan He [1] [2] [3] [4] and employed by scientists and engineers [5] [6] [7] to solve many problems in engineering and science. And it has proved tremendously effective to solve these problems. In this letter, we use the coupling of homotopy perturbation method and Laplace transform named homotopy transform method (HPTM) [8]-[13] to compare the rate of convergent to the solution with standard homotopy perturbation method.

\section{Homotopy Perturbation Method}

To illustrate the basic ideas of the (HPM), we consider the following nonlinear differential equation 


$$
L(u)+\mathrm{N}(u)-f(r)=0
$$

With the boundary conditions

$$
B\left(u, \frac{\partial u}{\partial n}\right)=0, \quad r \in \Gamma
$$

where $L$ is linear, while $\mathrm{N}$ is nonlinear, $f(r)$ is a known analytic function, $\Gamma$ is the boundary of the domain $\Omega$.

Define a homotopy $v(r, p): \Omega \times[0,1] \rightarrow R$ which satisfies

$$
\mathrm{H}(v, p)=(1-p)\left[L(v)-L\left(u_{0}\right)\right]+p[A(v)-f(r)]=0, p \in[0,1], r \in \Omega
$$

where $p \in[0,1]$ is an embedding parameter, $u_{0}$ is an initial approximation of Equation (3) then

$$
\begin{gathered}
\mathrm{H}(v, 0)=L(v)-L\left(u_{0}\right)=0, \\
\mathrm{H}(v, 1)=A(v)-f(r)=0
\end{gathered}
$$

The changing process of $p$ from zero to unity is just that of $v(r, p)$ from trivial solution $u_{0}(r)$ to original solution $u(r)$, in topology this is called deformation, $L(v)-L\left(u_{0}\right)$ and $A(v)-f(r)$ are called homotopic.

Here the imbedding parameter $p$ can be considered as "small parameter".

Assume that the solution of Equation (3) can be written as a power series in $p$

$$
v=v_{0}+p v_{1}+p^{2} v_{2}+\cdots
$$

Setting $p=1$ result in the approximate solution of Equation (6)

$$
u=\lim _{p \rightarrow 1} v=v_{0}+v_{1}+v_{2}+\cdots
$$

\section{Homotopy Perturbation Transform Method (HPTM)}

To illustrate the basic ideas of the (HPTM), we consider the following nonlinear differential equation with the initial conditions of the form

$$
\begin{gathered}
D u(x, t)+R u(x, t)+\mathrm{N} u(x, t)=g(x, t) \\
u(x, 0)=h(x), u_{t}(x, 0)=f(x) .
\end{gathered}
$$

where $D$ is the second order linear differential operator $D=\frac{\partial^{2}}{\partial t^{2}}, R$ is the linear differential operator of less order than $D$; $\mathrm{N}$ represents the general nonlinear differential operator and $g(x, t)$ is the source term.

Taking the Laplace transform (denoted by $L$ ) on both sides of Equation (8):

$$
\begin{gathered}
L[D u(x, t)]+L[R u(x, t)]+L[\mathrm{~N} u(x, t)]=L[g(x, t)] . \\
s^{2} L[u(x, t)]-s u(x, 0)-u_{t}(x, 0)+L[R u(x, t)]+L[\mathrm{~N} u(x, t)]=L[g(x, t)] .
\end{gathered}
$$

Using the initial conditions:

$$
L[u(x, t)]=\frac{h(x)}{s}+\frac{f(x)}{s^{2}}+\frac{1}{s^{2}} L[g(x, t)]-\frac{1}{s^{2}} L[R u(x, t)]-\frac{1}{s^{2}} L[\mathrm{~N} u(x, t)] .
$$


Operating with Laplace inverse on both sides of Equation (12) gives

$$
u(x, t)=G(x, t)-L^{-1}\left[\frac{1}{s^{2}} L[R u(x, t)+\mathrm{N} u(x, t)]\right] .
$$

where $G(x, t)$ represents the term arising from the source term and the prescribed initial conditions. Now we apply the HPM

$$
u(x, t)=\sum_{n=0}^{\infty} p^{n} u_{n}(x, t)
$$

And the nonlinear term can be decomposed as

$$
\mathrm{Nu}(x, t)=\sum_{n=0}^{\infty} p^{n} \mathrm{H}_{n}(u)
$$

where $\mathrm{H}_{n}(u)$ are He's polynomials given by

$$
\mathrm{H}_{n}\left(u_{0}, u_{1}, \cdots, u_{n}\right)=\left(\frac{1}{n !} \frac{\partial^{n}}{\partial p^{n}} \mathrm{~N}\left(\sum_{i=0}^{\infty} p^{i} u_{i}\right)\right)_{p=0}, \quad n=0,1,2, \cdots
$$

Substituting Equations (14) and (15) in Equation (13), we get

$$
\begin{aligned}
& \sum_{n=0}^{\infty} u_{n}(x, t) \\
& =G(x, t)-p\left(L^{-1}\left[\frac{1}{s^{2}} L\left[R \sum_{n=0}^{\infty} p^{n} u_{n}(x, t)+\sum_{n=0}^{\infty} p^{n} \mathrm{H}_{n}(u)\right]\right]\right) .
\end{aligned}
$$

Comparing the coefficient of like powers of $p$, the following approximations are obtained.

$$
\begin{aligned}
& p^{0}: u_{0}(x, t)=G(x, t), \\
& p^{1}: u_{1}(x, t)=-L^{-1}\left[\frac{1}{s^{2}} L\left[R u_{0}(x, t)+H_{0}(u)\right],\right. \\
& p^{2}: u_{2}(x, t)=-L^{-1}\left[\frac{1}{s^{2}} L\left[R u_{1}(x, t)+H_{1}(u)\right]\right], \\
& p^{3}: u_{3}(x, t)=-L^{-1}\left[\frac{1}{s^{2}} L\left[R u_{2}(x, t)+H_{2}(u)\right]\right],
\end{aligned}
$$

And so on.

\section{Application}

Example 1. Consider the inhomogeneous Advection problem [14]

$$
\frac{\partial u}{\partial t}+u \frac{\partial u}{\partial t}=-\sin (x+t)-\frac{1}{2} \sin 2(x+t), u(x, 0)=\cos x
$$

Standard HPM: According to homotopy Equation (3) we have

$$
\frac{\partial v}{\partial t}-\frac{\partial u_{0}}{\partial t}+p\left(v \frac{\partial v}{\partial x}+\frac{\partial u_{0}}{\partial t}+\sin (x+t)+\frac{1}{2} \sin 2(x+t)\right)=0
$$

And the solution for first few steps reads: 


$$
\begin{aligned}
v_{0}= & \cos x, \\
v_{1}= & \frac{1}{2} t \sin 2 x+\cos (x+t)-\cos x+\frac{1}{4} \cos 2(x+t)-\frac{1}{4} \cos 2 x, \\
v_{2}= & -\frac{1}{4} t^{2} \sin x \sin 2 x+\frac{1}{2} t^{2} \cos x \cos 2 x-\sin x \sin (x+t)+\sin ^{2} x \\
& +\cos x \cos (x+t)+\cos ^{2} x+t \sin 2 x-\frac{1}{8} \sin x \sin 2(x+t)+\frac{1}{8} \sin x \sin 2 x \\
& +\frac{1}{4} \cos x \cos 2(x+t)-\frac{1}{4} \cos x \cos 2 x+\frac{1}{4} t \sin x \cos 2 x+\frac{1}{2} t \cos x \sin 2 x \\
& \vdots
\end{aligned}
$$

Therefore, the approximate solution of Equation (19) can be written as

$$
\begin{aligned}
u= & \frac{1}{16}\left(\cos x-2 t^{2} \cos x+12 \cos 2 x+3 \cos 3 x-6 t^{2} \cos 3 x\right. \\
& +16 \cos (x+t)-\cos (2 t+x)+16 \cos (2 x+4)+4 \cos (2 x+2 t) \\
& -3 \cos (3 x+2 t)-2 t \sin x-8 t \sin 2 x-6 t \sin 3 x)+\cdots
\end{aligned}
$$

HPTM: to solve Equation (18) by MPTM, taking the Laplace transform on the both sides, subject to the initial condition, we get

$$
u(x, s)=\frac{\cos x}{s}+\frac{1}{s}\left[\left(\frac{-\cos x-s \sin x}{1+s^{2}}\right)-\frac{1}{2}\left(\frac{2 \cos 2 x+s \sin 2 x}{4+s^{2}}\right)\right]-\frac{1}{s} L\left[u \frac{\partial u}{\partial x}\right]
$$

Taking inverse Laplace transform, we get

$$
u(x, t)=\cos (x+t)+\frac{1}{4} \cos 2(x+t)-\frac{1}{4} \cos 2 x-L^{-1}\left[\frac{1}{s} L\left[u \frac{\partial u}{\partial x}\right]\right]
$$

Now, we apply the homotopy perturbation method; we have

$$
\begin{gathered}
u(x, t)=\sum_{n=0}^{\infty} p^{n} u_{n}(x, t) \\
\sum_{n=0}^{\infty} p^{n} u_{n}(x, t)=\cos (x+t)+\frac{1}{4} \cos 2(x+t)-\frac{1}{4} \cos 2 x-p\left(L^{-1}\left[\frac{1}{s} L\left[\sum_{n=0}^{\infty} p^{n} \mathrm{H}_{n}\right]\right]\right)
\end{gathered}
$$

where $H_{n}$ are He's polynomials that represent the nonlinear terms.

The first few components of He's polynomials, for example, are given by

$$
\begin{aligned}
& \mathrm{H}_{0}(u)=u_{0} u_{0 x}, \\
& \mathrm{H}_{1}(u)=u_{0} u_{1 x}+u_{1} u_{0 x},
\end{aligned}
$$

Comparing the coefficient of like powers of $p$, we have

$$
\begin{gathered}
p^{0}: u_{0}(x, t)=\cos (x+t)+\frac{1}{4} \cos 2(x+t)-\frac{1}{4} \cos 2 x, \\
p^{1}: u_{1}(x, t)=-L^{-1}\left[\frac{1}{s} L\left[\mathrm{H}_{0}(u)\right]\right]=-\frac{1}{4} \cos 2(x+t)+\frac{1}{4} \cos x+\frac{1}{64} \cos 4 x+\cdots,
\end{gathered}
$$

It is important to recall here that the noise terms appear between the components $u_{0}(x, t)$ and $u_{1}(x, t)$, more precisely, the noise terms 
$\pm \frac{1}{4} \cos 2(x+t) \pm \frac{1}{4} \cos 2 x$ between the components $u_{0}(x, t)$ and $u_{1}(x, t)$ can be cancelled and the remaining terms of $u_{0}(x, t)$ still satisfy the equation.

The exact solution is therefore

$$
u(x, t)=\cos (x+t)
$$

Example 2. Consider the inhomogeneous non-linear Klein Gordon equation [15]

$$
\frac{\partial^{2} u}{\partial t^{2}}-\frac{\partial^{2} u}{\partial x^{2}}+u^{2}=-x \cos t+x^{2} \cos ^{2} t
$$

Subject to the initial conditions

$$
u(x, 0)=x, \frac{\partial u}{\partial t}(x, 0)=0
$$

Standard HPM: According to homotopy Equation (3) we have

$$
\frac{\partial v^{2}}{\partial t^{2}}-\frac{\partial^{2} u_{0}}{\partial t^{2}}+p\left(\frac{\partial^{2} u_{0}}{\partial x^{2}}-\frac{\partial^{2} v}{\partial x^{2}}+v^{2}+x \cos t-x^{2} \cos ^{2} t\right)=0
$$

And the solution for first few steps reads:

$$
\begin{aligned}
v_{0}= & x \\
v_{1}= & -x+\frac{1}{8} x^{2}-\frac{3}{4} x^{2} t^{2}+x \cos t-\frac{1}{8} x^{2} \cos 2 x \\
v_{2}= & -\frac{1}{16} x^{2}+\frac{1}{8} t^{2}-\frac{t^{4}}{24}-2 x^{2}+t^{2} x^{2}+\frac{x^{3}}{16}-\frac{t^{2} x^{3}}{8}+\frac{t^{4} x^{3}}{24}+2 x^{2} \cos t \\
& -\frac{1}{16} \cos 2 x-\frac{1}{16} x^{3} \cos 2 x \\
& .
\end{aligned}
$$

Therefore, the approximate solution of Equation (26) with conditions (27) can be written as

$$
\begin{aligned}
u(x, t)= & -\frac{31}{16} x^{2}-\frac{3}{4} x^{2} t^{2}+\frac{1}{8} t^{2}-\frac{t^{4}}{24}+t^{2} x^{2}+\frac{x^{3}}{16}-\frac{t^{2} x^{3}}{8}+\frac{t^{4} x^{3}}{24}+x \cos t \\
& -\frac{1}{8} x^{2} \cos 2 x+2 x^{2} \cos t-\frac{1}{16} \cos 2 x-\frac{1}{16} x^{3} \cos 2 x+\cdots
\end{aligned}
$$

HPTM: To solve Equation (26) by MPTM, taking the Laplace transform on the both sides, subject to the initial condition, we get

$$
u(x, s)=\frac{x}{s}-\frac{x}{s\left(1+s^{2}\right)}+\frac{\left(2+s^{2}\right) x^{2}}{s^{3}\left(4+s^{2}\right)}+\frac{1}{s} L\left[\frac{\partial^{2} u}{\partial x^{2}}-u^{2}\right]
$$

Taking inverse Laplace transform, we get

$$
u(x, t)=x \cos t-\frac{1}{8} x^{2} \cos t+\frac{x^{2} t^{2}}{4}+\frac{x^{2}}{8}+L^{-1}\left[\frac{1}{s} L\left[\frac{\partial^{2} u}{\partial x^{2}}-u\right]\right]
$$

Now, we apply the homotopy perturbation method; we have 


$$
\begin{aligned}
\sum_{n=0}^{\infty} p^{n} u_{n}(x, t)= & x \cos t-\frac{1}{8} x^{2} \cos t+\frac{x^{2} t^{2}}{4}+\frac{x^{2}}{8} \\
& +p\left(L^{-1}\left[\frac{1}{s} L\left[\frac{\partial^{2}}{\partial x^{2}} \sum_{n=0}^{\infty} p^{n} u_{n}(x, t)-\left[\sum_{n=0}^{\infty} p^{n} \mathrm{H}_{n}\right]\right]\right]\right)
\end{aligned}
$$

The first few components of He's polynomials, for example, are given by

$$
\begin{aligned}
& \mathrm{H}_{0}(u)=u_{0}^{2}, \\
& \mathrm{H}_{1}(u)=2 u_{0} u_{1},
\end{aligned}
$$

Comparing the coefficient of like powers of $p$, we have $p^{0}: u_{0}(x, t)=x \cos t-\frac{1}{8} x^{2} \cos t+\frac{x^{2} t^{2}}{4}+\frac{x^{2}}{8}$, $p^{1}: u_{1}(x, t)=L^{-1}\left[\frac{1}{s} L\left[\frac{\partial^{2} u_{0}}{\partial x^{2}}-\mathrm{H}_{0}(u)\right]\right]=\frac{1}{8} x^{2} \cos t-\frac{x^{2} t^{2}}{4}-\frac{x^{2}}{8}+\frac{1}{64} x^{4} \cos 2 t+\cdots$

The noise terms $\pm \frac{1}{8} x^{2} \cos t \pm \frac{x^{2} t^{2}}{4} \pm \frac{x^{2}}{8}$ between the components $u_{0}(x, t)$ and $u_{1}(x, t)$ can be cancelled and the remaining terms of $u_{0}(x, t)$ still satisfy the equation.

The exact solution is therefore

$$
u(x, t)=x \cos t
$$

Example 3. Consider the following non homogeneous nonlinear PDE [16]:

$$
\frac{\partial^{2} u}{\partial t^{2}}+\frac{\partial^{2} u}{\partial x^{2}}+\left(\frac{\partial u}{\partial x}\right)^{2}=2 x+t^{4}
$$

With the initial conditions

$$
u(x, 0)=0, \frac{\partial u}{\partial t}(x, 0)=a,
$$

Standard HPM: According to homotopy perturbation method we have:

$$
\frac{\partial v^{2}}{\partial t^{2}}-\frac{\partial^{2} u_{0}}{\partial t^{2}}+p\left(\frac{\partial^{2} v}{\partial x^{2}}+\left(\frac{\partial v}{\partial x}\right)^{2}+\frac{\partial^{2} u_{0}}{\partial x^{2}}-2 x+t^{4}\right)=0
$$

Let's ignore the first few steps and start from determining $v_{i}$

$$
\begin{aligned}
v_{0} & =a t, \\
v_{1} & =x t^{2}+\frac{1}{30} t^{6}, \\
v_{2} & =0, \\
v_{3} & =\frac{1}{30} t^{6}, \\
& \vdots \\
v_{k} & =0, \quad k \geq 4
\end{aligned}
$$

Therefore, we obtain

$$
v_{0}=v_{0}+v_{1}+v_{2}+v_{3}+=a t+x t^{2} .
$$


HPTM: To solve Equation (34) by MPTM, taking the Laplace transform on the both sides, subject to the initial condition, we get

$$
u(x, s)=\frac{a}{s^{2}}+\frac{2 x}{s^{3}}+\frac{4 !}{s^{7}}-\frac{1}{s^{2}} L\left[\frac{\partial^{2} u}{\partial x^{2}}+\left(\frac{\partial u}{\partial x}\right)^{2}\right]
$$

Taking inverse Laplace transform, we get

$$
u(x, t)=a t+x t^{2}+\frac{t^{6}}{30}+L^{-1}\left[\frac{1}{s^{2}} L\left[\frac{\partial^{2} u}{\partial x^{2}}+\left(\frac{\partial u}{\partial x}\right)^{2}\right]\right]
$$

Now, we apply the homotopy perturbation method; we have

$$
\sum_{n=0}^{\infty} p^{n} u_{n}(x, t)=a t+x t^{2}+\frac{t^{6}}{30}-p\left(L^{-1}\left[\frac{1}{s^{2}} L\left[\frac{\partial^{2} u}{\partial x^{2}}+\sum_{n=0}^{\infty} p^{n} \mathrm{H}_{n}(u)\right]\right]\right)
$$

The first few components of He's polynomials, for example, are given by

$$
\begin{aligned}
& \mathrm{H}_{0}(u)=\left(\frac{\partial u_{0}}{\partial x}\right)^{2}=t^{4}, \\
& \mathrm{H}_{1}(u)=2 \frac{\partial u_{0}}{\partial x} \frac{\partial u_{1}}{\partial x}=0, \\
& \mathrm{H}_{2}(u)=\left(\frac{\partial u_{1}}{\partial x}\right)^{2}+2 \frac{\partial u_{0}}{\partial x} \frac{\partial u_{2}}{\partial x}=0,
\end{aligned}
$$

Comparing the coefficient of like powers of $p$, we have

$$
\begin{gathered}
p^{0}: u_{0}(x, t)=a t+x t^{2}+\frac{t^{6}}{30} \\
p^{1}: u_{1}(x, t)=-L^{-1}\left[\frac{1}{s^{2}}\left(L\left[\frac{\partial^{2} u_{0}}{\partial x^{2}}\right]+L\left[\mathrm{H}_{0}(u)\right]\right)\right]=-\frac{t^{6}}{30}, \\
p^{2}: u_{2}(x, t)=L^{-1}\left[\frac{1}{s}\left(L\left[\frac{\partial^{2} u_{1}}{\partial x^{2}}\right]+L\left[\mathrm{H}_{1}(u)\right]\right)\right]=0 \\
\vdots \quad u_{k}(x, t)=0, \quad k \geq 2
\end{gathered}
$$

Therefore, the exact solution is given by

$$
u(x, t)=a t+x t^{2}
$$

\section{Conclusion}

In this work, we compared HPTM with standard HPM, it is clear that the rate of convergence of HPTM is faster than HPM. In most cases, the number of calculations in the HPTM is less than HPM. Furthermore, the exact solution can easily be obtained by using HPTM in comparison to HPM in some equations. The HPTM usually results in the exact solution for the inhomogeneous problem, even for the problem which HPM leads to an approximate solution.

\section{References}

[1] He, J.-H. (1999) Homotopy Perturbation Technique. Computer Methods in Applied 
Mechanics and Engineering, 178, 257-262.

https://doi.org/10.1016/S0045-7825(99)00018-3

[2] He, J.-H. (2000) A Coupling Method of a Homotopy Technique and a Perturbation Technique for Non-Linear Problems. International Journal of Non-Linear Mechanics, 35, 37-43. https://doi.org/10.1016/S0020-7462(98)00085-7

[3] He, J.-H. (2003) Homotopy Perturbation Method: A New Nonlinear Analytical Technique. Applied Mathematics and Computation, 135, 73-79. https://doi.org/10.1016/S0096-3003(01)00312-5

[4] He, J.-H. (2009) An Elementary Introduction to the Homotopy Perturbation Method. Computers and Mathematics with Applications, 57, 410-412. https://doi.org/10.1016/j.camwa.2008.06.003

[5] Biazara, J. and Ghazvini, H. (2008) Homotopy Perturbation Method for Solving Hyperbolic Partial Differential Equations. Computers and Mathematics with Applications, 56, 453-458. https://doi.org/10.1016/j.camwa.2007.10.032

[6] Yldrm, A. (2009) Application of He's Homotopy Perturbation Method for Solving the Cauchy Reaction Diffusion Problem. Computers and Mathematics with Applications, 57, 612-616. https://doi.org/10.1016/j.camwa.2008.11.003

[7] Ghorbani, A. and Saberi-Nadjafi, J. (2007) He's Homotopy Perturbation Method for Calculating Adomian Polynomials. International Journal of Nonlinear Sciences and Numerical Simulation, 8, 229-232. https://doi.org/10.1515/IJNSNS.2007.8.2.229

[8] Khuri, S.A. (2001) A Laplace Decomposition Algorithm Applied to a Class of Nonlinear Partial Differential Equations. Journal of Applied Mathematics, 1, 141-155. https://doi.org/10.1155/S1110757X01000183

[9] Khan, M. and Hussain, M. (2011) Application of Laplace Decomposition Method on Semi-Infinite Domain. Numerical Algorithms, 56, 211-218.

https://doi.org/10.1007/s11075-010-9382-0

[10] Khan, M., Gondal, M.A. and Kumar, S. (2012) A New Analytical Solution Procedure for Nonlinear Integral Equations. Mathematical and Computer Modelling, 55, 1892-1897. https://doi.org/10.1016/j.mcm.2011.11.044

[11] Khan, Y. and Wu, Q. (2011) Homotopy Perturbation Transform Method for Solving Nonlinear Equations Using He's Polynomials. Computers \& Mathematics with Applications, 61, 1963-1967. https://doi.org/10.1016/j.camwa.2010.08.022

[12] Mishr, H.M. and Nagar, A.K. (2012) He-Laplace Method for Linear and Nonlinear Partial Differential Equations. Journal of Applied Mathematics, 2012, Article ID 180315.

[13] Kumar, J.S.D. and Rathore, S. (2012) Application of Homotopy Perturbation Transform Method for Solving Linear and Nonlinear Klein-Gordon Equations. Journal of Information and Computing Science, 7, 131-139.

[14] Wazwaz, A.M. (2009) Partial Differential Equations and Solitary Waves Theory, Nonlinear Physical Science. Springer, New York.

https://doi.org/10.1007/978-3-642-00251-9

[15] Chowdhury, M.S.H. and Hashim, I. (2009) Application of Homotopy-Perturbation Method, to Klein-Gordon and Sine-Gordon Equations. Chaos, Solitons and Fractals, 39, 1928-1935. https://doi.org/10.1016/j.chaos.2007.06.091

[16] Gupta, S., Kumar, D. and Singh, J. (2015) Analytical Solutions of Convection-Diffusion Problems by Combining Laplace Transform Method and Homotopy Perturbation Method. Alexandria Engineering Journal, 54, 645-651. https://doi.org/10.1016/j.aej.2015.05.004 